\title{
Influenza A virus and p53: Can the Two Walk Together? A Commentary on "p53 and the Viral Connection: Back into the Future"
}

\author{
Ronit Aloni-Grinstein ${ }^{1,2 \#}$, Yan Stein ${ }^{1 \#}$ and Varda Rotter ${ }^{1 *}$ \\ 'Department of Molecular Cell Biology, Weizmann Institute of Science, Rehovot 7610001, Israel \\ ${ }^{2}$ Department of Biochemistry and Molecular Genetics, Israel Institute for Biological Research, Ness-Ziona, Israel \\ \#These authors contributed equally to this manuscript
}

\section{Article Info}

\section{Article Notes}

Received: December 19, 2019

Accepted: January 22, 2020

\section{*Correspondence:}

Prof. Varda Rotter, Department of Molecular Cell Biology, Weizmann Institute of Science, Rehovot 7610001, Israel; Telephone No: 972-8-9344501

Email: varda.rotter@weizmann.ac.il

C 2020 Rotter V. This article is distributed under the terms of the Creative Commons Attribution 4.0 International License.

\section{Keywords}

Influenza

Respiratory infections

p53

Antiviral effects

Immune regulation

Apoptosis

\section{Abstract}

Influenza $A$ is a very common cause for respiratory infections, which constitutes a major public health concern due to high rates of morbidity and mortality in high-risk population. In our previous publication, 'p53 and the Viral Connection: Back Into the Future', we discussed the involvement of the p53 tumor suppressor in the response to non-tumorigenic viruses, among which is the Influenza virus. In the current comment, we focus on the interplay between $\mathrm{p} 53$ and the influenza viral cycle. We discuss recent publications that provide evidence for the potential antiviral and pro-viral roles of p53 and its isoforms towards influenza, both in the host cell, as well as in the immune system. On the other side of the coin, we also discuss how the influenza virus may manipulate $\mathrm{p} 53$ to promote its own replication and spreading. An understanding of the interplay between p53 and the virus may lead to the development of a host-based influenza virus therapy.

Influenza A virus (IAV) is one of the most common sources of human respiratory infections, causing morbidity and death in highrisk population and constituting a major public health and economic concern. Up to $20 \%$ of the world population may be affected by an IAV infection, and up to 250,000-500,000 deaths are reported each year ${ }^{1}$. As a preventive strategy, prophylactic vaccination is offered to high-risk population. However, as IAV possesses high rates of genetic drift and genetic shift, new IAV strains continuously arise, making previously prepared vaccines and acquired immunity less relevant $t^{2}$. Thus, annual development of new vaccines is compulsory. Consequently, efforts are made to develop a broad universal vaccine ${ }^{3}$. Similarly, more and more resistant strains of IAV arise against the M2 and the NA inhibitors, the two classes of FDA approved antiIAV drugs, pointing to the urgent need for the development of new therapies ${ }^{4}$. These efforts are based on targeting conserved domains of the viral protein or, alternatively, targeting host factors that are involved in the viral life cycle.

In our recently published review "p53 and the viral connection: Back into the future" ${ }^{\prime 5}$, we highlighted the roles of the tumor suppressor p53, known as "the guardian of the genome", in tumor virology. In a historical perspective view of 40 years of research, we describe the different strategies by which tumor-promoting viruses manipulate wild-type p53 (WTp53) functions in ways that may seed the onset of cancer development. Alongside, through the understanding of the tactics by which various viruses hijack WTp53 functions to their own benefit, came the appreciation of the roles of WTp53 also in the life cycle of non-oncogenic viruses, such as the 
IAV, the poxvirus (smallpox and vaccinia viruses) ${ }^{6,7}$, the flavivirus (Zika and West Nile) ${ }^{8,9}$, Japanese encephalitis virus ${ }^{10}$, Human Immunodeficiency virus (HIV) type $\mathrm{I}^{11,12}$, Human herpes simplex virus $1^{13,14}$ and more.

One of the hallmarks of IAV infection is the modulation in WTp53 levels and activity. Indeed, various studies reported different mechanisms by which IAV infection modulates WTp53 activity, resulting in different biological outcomes, of which most launch antiviral effects ${ }^{15-18}$, while some favor IAV replication ${ }^{19,20}$. Terrier et al. reported a biphasic modulation of p53 transcriptional activity during IAV infection, with a high activity at initial time points, followed by a marked reduction during the advanced stages $^{21}$. Moreover, global transcriptome analyses by microarray showed that p53 is implicated in the response to IAV infection, both in vitro ${ }^{20,22}$, as well as in vivo ${ }^{18}$.

WTp53 does not play an active role in initial virus entry. However, as infection proceeds and cell stress increases, apoptosis is induced and type I interferon pathways are activated, leading to enhanced innate and adaptive immune responses as an outcome of WTp53 activity ${ }^{15,16,18}$. Indeed, Shen et al. ${ }^{23}$ reported on a biphasic pattern of WTp53 elevation, where the first elevation in p53 levels occurs upon IAV adsorption, prior to the onset of virus replication, whereas the second elevation occurs at the middle-late phase of IAV replication. Of note, this elevation in p53 level does not necessarily correlate with p53 activity, as it was shown that some p53 target genes were actually downregulated at late time points, an effect which was mediated by the viral protein $\mathrm{NS}^{21}$. It is possible, however, that some genes, such as immune modulating genes, are upregulated, while another subset of p53 target genes is downregulated. Aside from p53induced immune response, p53-mediated apoptosis may also be regarded as a host defense mechanism against the virus $^{17}$. Altogether, these actions are aimed at reducing infectivity. However, it should be noted that appropriately timed apoptosis benefits IAV and has been reported to be central for viral replication and spreading ${ }^{24}$. Consistently with this finding, Wang et al. reported that expression of pro-apoptotic molecules, including p53, was associated with increased viral titers in lung cells upon IAV H1N1 infection ${ }^{19}$. Thus, the conflicting role of p53 in either facilitating or attenuating IAV replication may stem from the opposed roles reported for apoptosis for IAV infection. Interestingly, Wang et al. ${ }^{20}$ report contrasting biological effects of different p53 forms. p53 was shown to downregulate, independently of its transcriptional activity, the antiviral host factors interferon-induced transmembrane family members IFITM1, IFITM2 and IFITM3, which are known to restrict IAV infectivity, thus extending the time window of efficient infection and promoting infectivity ${ }^{20}$. In their study, the authors used specific CRISPR/Cas9 knockout (KO) in the influenza host cells, which caused either the entire loss of p53 protein, or the loss of the p53 full-length protein, while retaining the expression of the $\Delta 40$ p53 isoform. Interestingly, the entire p53 KO caused a marked decrease in the efficiency of influenza viral replication, while retaining the expression of the $\Delta 40$ isoform caused a significant increase, compared to WT host cells, in viral replication efficiency. These effects were mediated by affecting the expression levels of antiviral host factors interferon-induced transmembrane family members IFITM1, IFITM2 and IFITM3, which were upregulated upon entire p53 KO, but reduced upon retention of the $\Delta 40$ isoform. In all, these results suggest that while full-length WTp53 limits influenza replication, the $\Delta 40$ isoform seems to promote it, at least in part by modulating the host cell antiviral response. Intriguingly, another report suggested that the NS1 protein of the influenza virus manipulates the host splicing machinery, leading to increased expression of $\mathrm{p} 53 \beta$ and $\gamma$ isoforms and to increased viral replication ${ }^{25}$. In addition to modulating p53 isoform expression, viral proteins may also modulate p53 stability and protein accumulation via interacting with p53 itself. For example, the viral nucleoprotein (NP) associates with $\mathrm{p} 53^{26}$, thus impairing the binding of $\mathrm{p} 53$ to $\mathrm{Mdm} 2$, the major factor that regulates p53 ubiquitination and degradation ${ }^{27}$. In turn, this inhibition of p53-Mdm2 interaction by NP contributes to p53 accumulation, stabilization and to an increase in its transcriptional activity $^{26}$. It seems therefore, that p53 transcriptional activity is modulated during the course of infection, with a significant increase at early stages due to the presence of NP, and a significant decrease during the late stages of infection, where the levels of NS1 rise. Altogether, these findings imply an ongoing "arms race" between the host p53 and the influenza virus, in which p53, in all, aims to limit viral replication, while the virus aims to modulate p53 activity, in part by modulating the expression of p53 isoforms, or by modulating p53 stability and activity.

Aside from the role of WTp53 in the host cell, another aspect that is worth considering regarding the effect of WTp53 on IAV infectivity is the known role of WTp53 in the immune system ${ }^{28,29}$. Indeed, p53 KO mice exhibited impaired function of immune cells, including dendritic cells, monocytes and cytotoxic T-cells, as well as attenuated induction of cytokines, which led to increased IAV replication and, consequently, to increased mortality ${ }^{15}$. Interestingly, not only that p53 induces the expression of target genes in the immune system ${ }^{30}$, but the interferon pathway, which comprises some p53 target genes, regulates the transcription of p53 itself ${ }^{31}$, thus suggesting the existence of a positive feedback loop. Consistently with the aforementioned studies, it was shown that mice harboring an extra p53 copy ('super p53' mice) were more resistant to viral infection than their WT counterparts ${ }^{32}$. 
Thus, it is not only p53 cell-autonomous functions that exert an antiviral response, but p53 non-cell autonomous functions in the immune system are crucial for the host's antiviral response as well.

Taken together, it is intriguing to hope that a broader understanding of the interplay between p53 and IAV may open new horizons for the use of p53-based therapies (developed for cancer diseases) as general antiviral agents. A potential advantage of p53-based therapies to IAV may be the activity of p53 both at the prime site of IAV infection, at the epithelial cells, as well as in the immune cell subset involved in the antiviral response, resulting in a dual additive antiviral response. However, one must bear in mind the potential toxic adverse effects of p53 stabilization on normal cells, and perhaps try to ameliorate them by targeting or delivering the p53-based therapies specifically to virus-infected cells. Moreover, the use of natural products that were shown to inactivate IAV through p53 activation may be beneficial ${ }^{33}$.

\section{Acknowledgments}

Research in the laboratory of Varda Rotter is supported by a Center of Excellence Grant from the Israel Science Foundation (ISF) and a Center of Excellence Grant from the Flight Attendant Medical Research Institute (FAMRI). Varda Rotter is the incumbent of the Norman and Helen Asher Professorial Chair for Cancer Research at the Weizmann Institute.

\section{Conflicts of Interest}

The authors declare no conflict of interest.

\section{References}

1. Król E, Rychłowska M, SzewczykB. Antivirals--current trends in fighting influenza. Acta Biochim Pol. 2014; 61(3): 495-504.

2. van de Wakker SI, Fischer MJE, Oosting RS. New drug-strategies to tackle viral-host interactions for the treatment of influenza virus infections. Eur J Pharmacol. 2017; 809: 178-190.

3. Vemula SV, Sayedahmed EE, Sambhara S, et al. Vaccine approaches conferring cross-protection against influenza viruses. Expert Rev Vaccines. 2017; 16(11): 1141-1154.

4. Loregian A, Mercorelli B, Nannetti G, et al. Antiviral strategies against influenza virus: towards new therapeutic approaches. Cell Mol Life Sci. 2014; 71(19): 3659-3683.

5. Aloni-Grinstein R, Charni-Natan M, Solomon H et al. p53 and the Viral Connection: Back into the Future $\neq$. Cancers (Basel). 2018; 10(6): 178.

6. Wali A, Strayer DS. Infection with Vaccinia Virus Alters Regulation of Cell Cycle Progression. DNA Cell Biol. 1999; 18(11): 837-843.

7. Santos CR, Vega FM, Blanco $S$, et al. The vaccinia virus B1R kinase induces p53 downregulation by an Mdm2-dependent mechanism. Virology. 2004; 328(2): 254-265.

8. Ghouzzi V El, Bianchi FT, Molineris I, et al. ZIKA virus elicits P53 activation and genotoxic stress in human neural progenitors similar to mutations involved in severe forms of genetic microcephaly and p53. Cell Death Dis. 2016; 7(10): e2440-e2440.

9. Yang MR, Lee SR, Oh W, et al. West Nile virus capsid protein induces p53-mediated apoptosis via the sequestration of HDM2 to the nucleolus. Cell Microbiol. 2007; 10(1): 165-176.

10. Deng X, Wei J, Shi Z, et al. Tumor suppressor p53 functions as an essential antiviral molecule against Japanese encephalitis virus. J Genet Genomics. 2016; 43(12): 709-712.

11. Greenway AL, McPhee DA, Allen K, et al. Human immunodeficiency virus type $1 \mathrm{Nef}$ binds to tumor suppressor p53 and protects cells against p53-mediated apoptosis. J Virol. 2002; 76(6): 2692-2702.

12. Izumi T, Io K, Matsui M, et al. HIV-1 viral infectivity factor interacts with TP53 to induce G2 cell cycle arrest and positively regulate viral replication. Proc Natl Acad Sci U S A. 2010; 107(48): 20798-20803.

13. Maruzuru Y, Fujii H, Oyama M, et al. Roles of p53 in herpes simplex virus 1 replication. J Virol. 2013; 87(16): 9323-9332.

14. Maruzuru Y, Koyanagi N, Takemura N, et al. p53 Is a Host Cell Regulator during Herpes Simplex Encephalitis. J Virol. 2016; 90(15): 6738-6745.

15. Muñoz-Fontela C, Pazos M, Delgado I, et al. p53 Serves as a Host Antiviral Factor That Enhances Innate and Adaptive Immune Responses to Influenza A Virus. J Immunol. 2011; 187(12): 64286436.

16. Zhu Z, Yang Y, Wei J, et al. Type I interferon-mediated immune response against influenza A virus is attenuated in the absence of p53. Biochem Biophys Res Commun. 2014; 454(1): 189-195.

17. Turpin E, Luke K, Jones J, et al. Influenza Virus Infection Increases p53 Activity: Role of p53 in Cell Death and Viral Replication. J Virol. 2005; 79(14): 8802-8811.

18. Yan W, Wei J, Deng X, et al. Transcriptional analysis of immune-related gene expression in p53-deficient mice with increased susceptibility to influenza A virus infection. BMC Med Genomics. 2015; 8(1): 52.

19. Wang X, Tan J, Zoueva O, et al. Novel pandemic influenza A (H1N1) virus infection modulates apoptotic pathways that impact its replication in A549 cells. Microbes Infect. 2014; 16(3): 178-186.

20. Wang B, Lam TH, Soh MK, et al. Influenza A Virus Facilitates Its Infectivity by Activating p53 to Inhibit the Expression of InterferonInduced Transmembrane Proteins. Front Immunol. 2018; 9: 1193.

21. Terrier 0, Diederichs A, Dubois J, et al. Influenza NS1 interacts with p53 and alters its binding to p53-responsive genes, in a promoterdependent manner. FEBS Lett. 2013; 587(18): 2965-2971.

22. Terrier O, Josset L, Textoris J, et al. Cellular transcriptional profiling in human lung epithelial cells infected by different subtypes of influenza A viruses reveals an overall down-regulation of the host $\mathrm{p} 53$ pathway. Virol J. 2011; 8(1): 285.

23. Shen $Y$, Wang $X$, Guo $L$, et al. Influenza A virus induces p53 accumulation in a biphasic pattern. Biochem Biophys Res Commun. 2009; 382(2): 331-335.

24. McLean JE, Datan E, Matassov D, et al. Lack of Bax prevents influenza A virus-induced apoptosis and causes diminished viral replication. J Virol. 2009; 83(16): 8233-8246.

25. Dubois J, Traversier A, Julien T, et al. The Nonstructural NS1 Protein of Influenza Viruses Modulates TP53 Splicing through Host Factor CPSF4. J Virol. 2019; 93(7): e02168-18.

26. Wang X, Deng X, Yan W, et al. Stabilization of p53 in influenza A virusinfected cells is associated with compromised MDM2-mediated ubiquitination of p53. J Biol Chem. 2012; 287(22): 18366-18375.

27. Haupt $Y$, Maya R, Kazaz A, et al. Mdm2 promotes the rapid degradation of p53. Nature. 1997; 387(6630): 296-299.

28. Muñoz-Fontela C, Mandinova A, Aaronson SA, et al. Emerging roles of p53 and other tumour-suppressor genes in immune regulation. Nat Rev Immunol. 2016; 16(12): 741-750. 
29. Cui Y, Guo G. Immunomodulatory Function of the Tumor Suppressor p53 in Host Immune Response and the Tumor Microenvironment. Int J Mol Sci. 2016; 17(11): 1942.

30. Muñoz-Fontela C, Macip S, Martínez-Sobrido L, et al. Transcriptional role of p53 in interferon-mediated antiviral immunity. J Exp Med. 2008; 205(8): 1929-1938.

31. Takaoka A, Hayakawa $S$, Yanai $H$, et al. Integration of interferon- $\alpha / \beta$ signalling to p53 responses in tumour suppression and antiviral defence. Nature. 2003; 424(6948): 516-523.

32. Mun̄oz-Fontela C, Angel Garcia M, Garcia-Cao I, et al. Resistance to viral infection of super p53 mice. Oncogene. 2005; 24(18): 3059-3062.

33. Khalil H, Abd El Maksoud AI, Roshdey T, et al. Guava flavonoid glycosides prevent influenza A virus infection via rescue of P53 activity. J Med Virol. 2019; 91(1): 45-55. 